\title{
RAPID PULSATIONS IN SUB-THz SOLAR BURSTS
}

\author{
Pierre Kaufmann $^{1,2}$, C. Guillermo Giménez de Castro ${ }^{1}$, Emilia Correia ${ }^{1,3}$, Joaquim E. R. Costa ${ }^{3}$, Jean-Pierre Raulin ${ }^{1}$, \\ AND Adriana Silva VÁlio ${ }^{1}$ \\ ${ }^{1}$ Centro de Rádio-Astronomia e Astrofísica Mackenzie, Escola de Engenharia, Universidade Presbiteriana Mackenzie, Rua Consolação 896, 01302-907 São Paulo, \\ SP, Brazil \\ ${ }^{2}$ Centro de Componentes Semicondutores, Universidade Estadual de Campinas, Cidade Universtária” Zeferino Vaz", 13981-970 Campinas, SP, Brazil \\ ${ }^{3}$ Instituto Nacional de Pesquisas Espaciais, Av. dos Astronautas 1758, 12227-010 São José dos Campos, SP, Brazil \\ Received 2008 December 18; accepted 2009 March 10; published 2009 May 1
}

\begin{abstract}
A new solar burst emission spectral component has been found showing sub-THz fluxes increasing with frequency, spectrally separated from the well known microwave component. Rapid pulsations are found present in all events observed at the two frequencies of the solar submillimeter-wave telescope: 212 and $405 \mathrm{GHz}$. They were studied in greater detail for three solar bursts exhibiting the new $\mathrm{THz}$ spectral component. The pulse amplitudes are of about $5 \%-8 \%$ of the mean flux throughout the bursts durations, being comparable for both frequencies. Pulsations range from one pulse every few seconds to $8-10$ per second. The pulse repetition rates $(R)$ are linearly proportional to the mean burst fluxes $(S)$, following the simple relationship $S=k R$, suggesting that the pulsations might be the response to discrete flare particle accelerator injections quantized in energy. Although this result is consistent with qualitative trends previously found in the $\mathrm{GHz}$ range, the pulse amplitude relative to the mean fluxes at the sub-THz frequencies appear to be nearly ten times smaller than expected from the extrapolation of the trends found in the $\mathrm{GHz}$ range. However there are difficulties to reconcile the nearly simultaneous $\mathrm{GHz}$ and $\mathrm{THz}$ burst emission spectrally separated components, exhibiting rapid pulsations with considerably larger relative intensities in the $\mathrm{GHz}$ range.
\end{abstract}

Key words: acceleration of particles - MHD - radiation mechanisms: non-thermal - Sun: flares - Sun: oscillations - Sun: radio radiation

\section{INTRODUCTION}

Solar flare impulsive emissions in the radio range are usually explained by two distinct processes: radiation at longer metricdecimetric wavelengths exhibit flux densities decreasing with frequency (usually narrow band), attributed to excited plasma emissions higher in the solar corona (Wild \& Smerd 1972). Broadband microwaves are attributed to gyro-synchrotron losses in the magnetic field while accelerated electrons move into denser regions to produce hard $\mathrm{X}$ - and gamma rays by collision losses (Dulk et al. 1979; Kundu \& Vlahos 1982; Bastian et al. 1998). The two mechanisms compose the well known U-shaped radio burst spectra (Castelli 1972; Nita et al. 2004). The $\mathrm{THz}$ flux versus frequency increasing spectral component, recently discovered by Kaufmann et al. (2004), suggests a "W-shaped" spectrum as illustrated in Figure 1, possibly representative of a third emission mechanism.

Time profiles at various radio wavelengths and X-ray ranges are not always well correlated. Examples of time profiles with delays of several seconds at short microwaves and hard X-rays (> $30 \mathrm{keV}$ ) were found (Takakura et al. 1983; Lim et al. 1992; Kaufmann et al. 1983; Costa \& Kaufmann 1983; Silva et al. 2000). The burst impulsive phase onset may occur first at lower frequencies, although there are also examples with delays in the opposite sense. Fast superimposed time structures at much shorter second to subsecond scales, obtained by observations with higher sensitivity and time resolution, have indicated that they are synchronized by less than $200 \mathrm{~ms}$ at short microwaves and at hard X-rays (Cornell et al. 1984; Takakura et al. 1983; Kaufmann et al. 2000).

Broadband solar burst rapid pulsations at $\mathrm{cm}-\mathrm{mm}$ wavelengths were well characterized (Butz et al. 1976; Gaizauskas \& Tapping 1980; Kaufmann et al. 1980, 1985; Allaart et al. 1990, Benz et al. 1992; Qin et al. 1996; Huang et al. 1996; Raulin et al. 1998; Nakajima 2000; Altyntsev et al. 2000). A puzzling qualitative proportionality between pulse repetition rates and underlying mean fluxes has been found at 10, 22, 30, 44, 48, and $90 \mathrm{GHz}$ (Kaufmann et al. 1980, 1985; Correia \& Kaufmann 1987; Raulin et al. 1998) and at 9.4 and 15 GHz (Qin et al. 1996; Huang et al. 1996). It might be interpreted as the response to discrete injections quasiquantized in energy (Kaufmann et al. 1980); or by wave-particle plasma instabilities', saturation time inversely proportional to radiation time (Huang et al. 1996); or by modulation of flare kernel emission by magnetic field oscillations driven by Alfvén waves which period decreases for larger temperatures (Qin et al. 1996).

The present study addresses the distinctive aspects of rapid pulsations observed at sub-THz solar bursts. They are analyzed for their repetition rates versus time profiles at sub- $\mathrm{THz}$ frequencies, at hard $\mathrm{X}$-rays and $\gamma$-rays and on the differences when compared to pulsations in the microwave range of frequencies.

\section{THE SUB-THz SOLAR BURST EMISSIONS}

The solar submillimeter telescope (SST) is operated at the El Leoncito Astronomical Complex (CASLEO), San Juan, Argentina Andes. It has four $212 \mathrm{GHz}$ and two $405 \mathrm{GHz}$ radiometers, placed at the focal plane of a radome-enclosed $1.5 \mathrm{~m}$ diameter Cassegrain antenna (Kaufmann et al. 2008). Three partial overlapping beams at $212 \mathrm{GHz}$ allow the determination of the accurate burst spatial position and the calculation of the flux density (Giménez de Castro et al. 1999). SST has found that rapid pulsations are common to all sub-THz events observed (Kaufmann et al. 2001; Makhmutov et al. 2003; Raulin et al. 2003). Furthermore, a new kind of burst emission was discovered exhibiting a $\mathrm{THz}$ component spectrally separated from the well known microwave component (Kaufmann et al. 2004). The T-rays component was found for other events (Silva et al. 2007; Cristiani et al. 2008; Kaufmann et al. 2009). In other 
No. 1, 2009

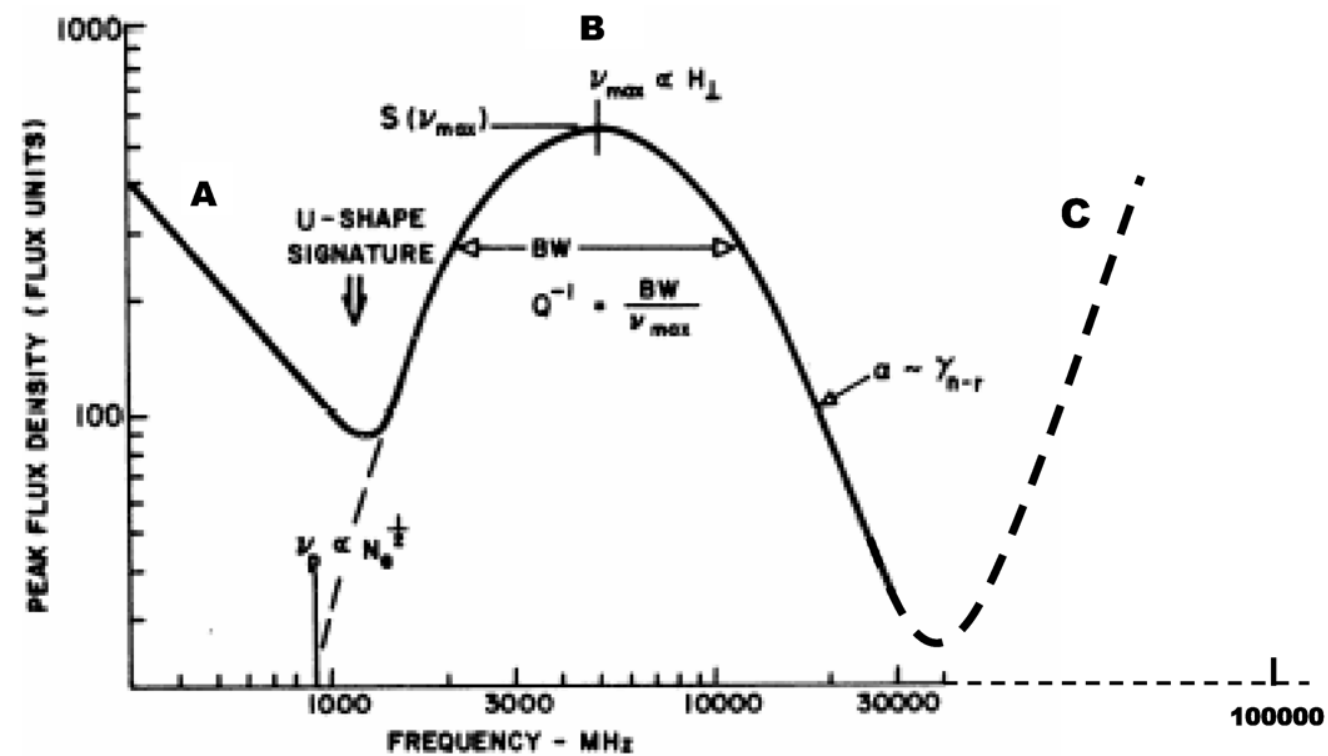

Figure 1. Schematic representation of solar radio burst emissions, from metric to submillimetric wavelengths, adapted from a plot given by Castelli (1972) for $\mathrm{U}$-shaped spectra (up to $30 \mathrm{GHz}$ ). The newly discovered THz component (Kaufmann et al. 2004) gives a more general W-shaped radio emission spectra buildup from three different emission mechanisms: A, B, and C.
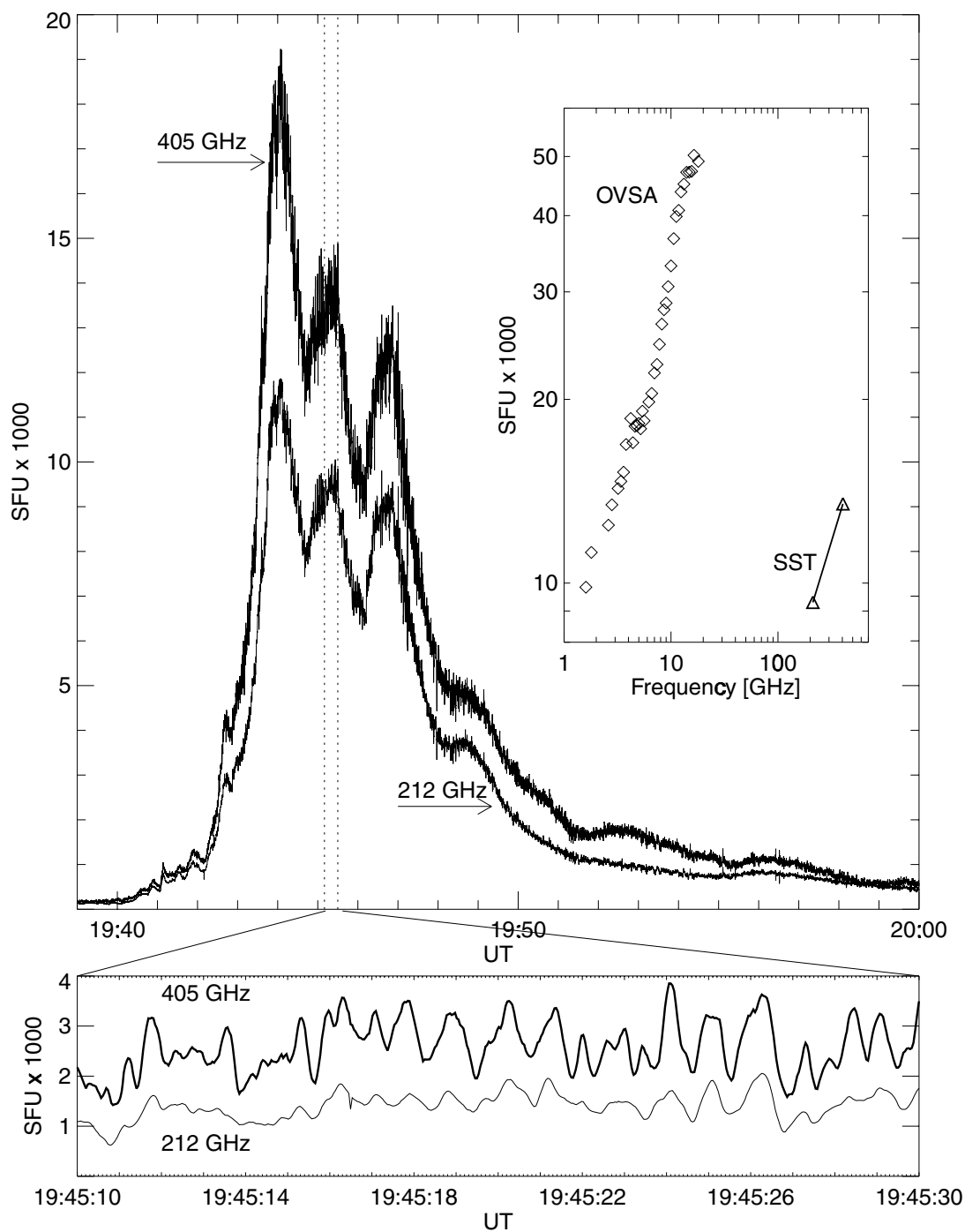

Figure 2. 2003 November 4 solar burst. Top panel shows the time profiles obtained by SST at sub-THz frequencies. The inlet shows the spectral trend for the middle temporal peak, evidencing two spectrally separated components, at microwaves and at sub-THz. Bottom panel exhibits a $20 \mathrm{~s}$ zoom of $40 \mathrm{~ms}$ data near the middle maximum structure showing the rapid superimposed pulsations. 


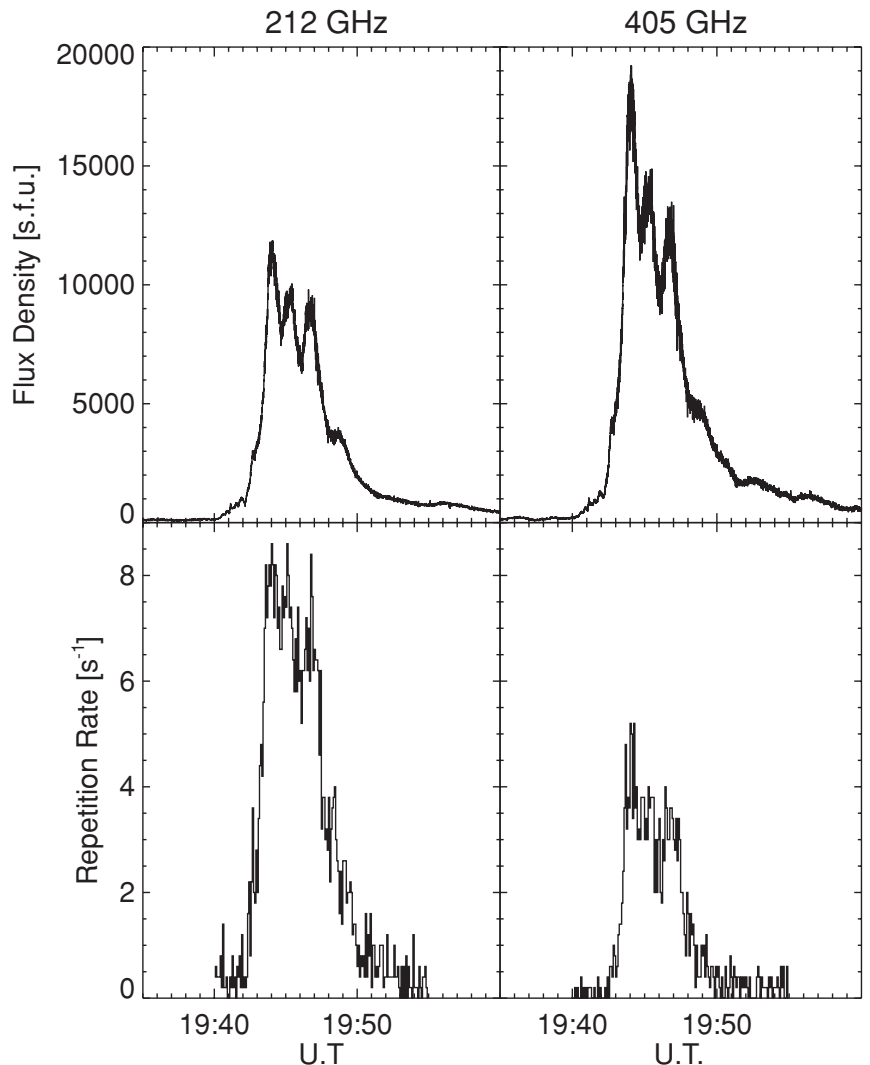

(a)

(b)

Figure 3. 2003 November 4 flare: (a) $212 \mathrm{GHz} 40 \mathrm{~ms}$ flux time profile (top), and pulse rate profile (bottom). (b) $405 \mathrm{GHz} 40 \mathrm{~ms}$ flux time profile (top), and pulse rate profile (bottom). Pulse rate obtained on $5 \mathrm{~ms}$ data, every $5 \mathrm{~s}$ interval, counted using wavelet decomposition.

examples, it appears as spectral frequency upturn trends during periods of certain events (Raulin et al. 2004; Lüthi et al. 2004).

The sub-THz time profiles and spectrum for the 2003 November 4 giant solar flare are shown in Figure 2. The spectrum shown at the inlet panel is for the middle major peak obtained with $\mathrm{GHz}$ data from Owens Valley Solar Array (OVSA) averaged over $30 \mathrm{~s}$, and SST data. It shows another spectral component, peaking somewhere in the $\mathrm{THz}$ range, separated from the well known microwaves component. It is also observed that subsecond pulsations are superimposed onto the underlying flux time profile. A $20 \mathrm{~s}$ zoom near the middle major peak of the $40 \mathrm{~ms}$ resolution data at the bottom of Figure 2 shows pulses with significant amplitudes, similar to the two frequencies repeating at a rate close to 2 per second. The amplitude of the pulses relative to the mean flux $(\Delta S / S)$ is of about $8 \%$ at 212 and $405 \mathrm{GHz}$, nearly all the time throughout the event duration. The pulses' amplitude are more than 10 times larger than the increase in system noise due to the burst contribution at the main peak, i.e., of about 5 and 9 solar flux units (SFUs), at 212 and $405 \mathrm{GHz}$, respectively (1 $\left.\mathrm{SFU}=10^{-22} \mathrm{~W} \mathrm{~m}^{-2} \mathrm{~Hz}^{-1}\right)$.

Pulse rate count throughout the burst duration was performed using a wavelet decomposition technique (Kurths \& Schwarz 1994; Aschwanden et al. 1998; Giménez de Castro et al. 2001; Makhmutov et al. 2003; Raulin et al. 2003). For this analysis, we use wavelets derived from a triangular mother wavelet, which are representative of the pulses we want to observe (Bendjoya et al. 1993). The use of wavelets in solar flare analysis is not new (Krüger et al. 1994; Aschwanden et al. 1998; Giménez de Castro et al. 2001; Makhmutov et al. 2003). The method is

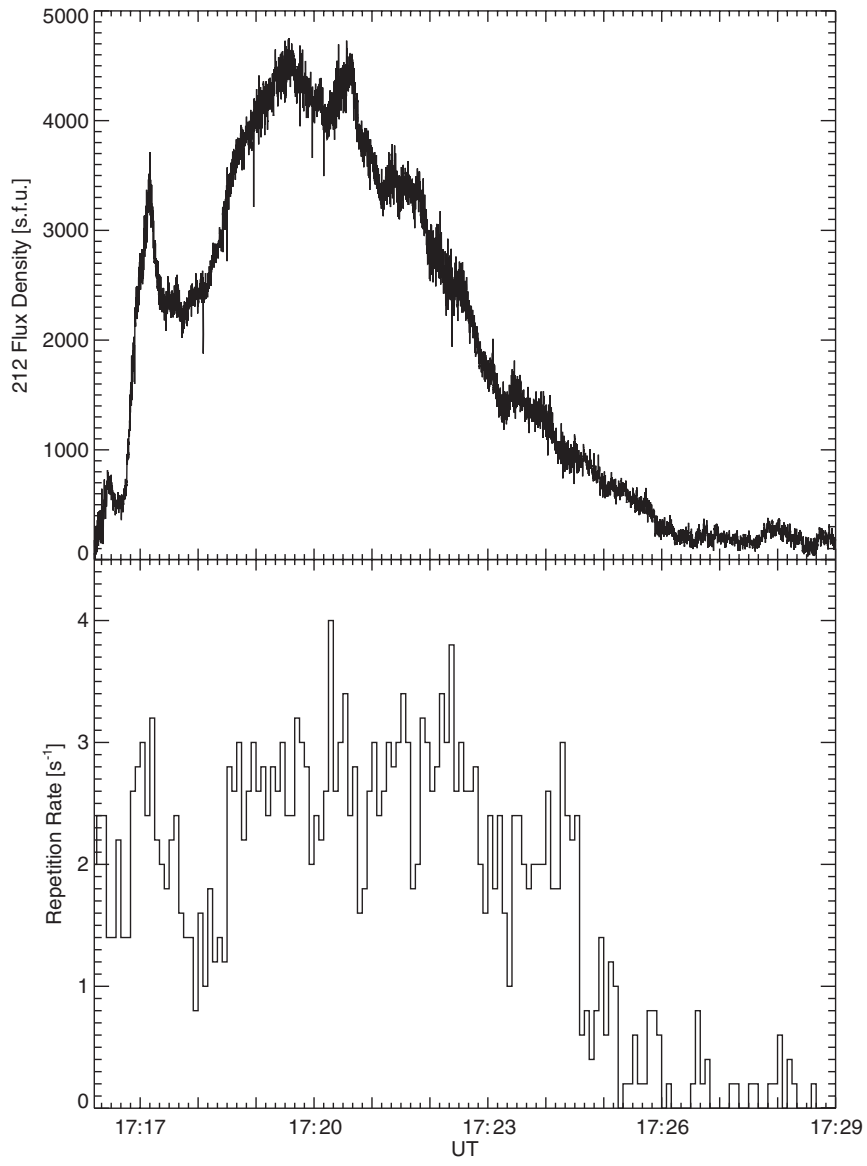

Figure 4. 2003 November 2 flare. The sub-THz data were considerably prejudiced by poor atmosphere transmission. Pulses at $405 \mathrm{GHz}$ were below the noise fluctuation threshold set prior to the event, and could not be counted. The start time was not well defined. We show here only the $212 \mathrm{GHz} 40 \mathrm{~ms}$ flux time profile (top), and pulse rate profile (bottom). Pulse rate obtained on $5 \mathrm{~ms}$ data, every $5 \mathrm{~s}$ interval, counted using wavelet decomposition.

useful to separate the original time series in different temporal scales. Every temporal scale is represented as a time series with 0 mean value; therefore, every significant local maximum can be considered a "pulse." As a requisite, pulses are counted when their intensity exceeds a noise fluctuation threshold level set before the burst onset. The analysis was performed using $5 \mathrm{~ms}$ resolution data. We used temporal scales ranging from 40 to $160 \mathrm{~ms}$ and defined a criterion to avoid counting the same pulse in two different scales. The method was tested on time intervals with actually counted pulses, as illustrated in Figure 2(a). The results for the flux time profiles and for the pulse repetition rates, for the three events studied here, are shown in Figures 3-5.

The identification of pulses superimposed onto the flux time profiles is strongly dependent on the quality of the atmospheric transmission. Transmission was extremely good for the 2003 November 4 solar burst observation, for which the time profiles were almost identical at 212 and $405 \mathrm{GHz}$ both for fluxes and for the repetition rates (Figures 2(a) and 3). Atmospheric transmission was particularly bad for the 2003 November 2 observations, for which the pulsations were detectable only at $212 \mathrm{GHz}$ (Figure 4). Poor transmission for the 2006 December 6 observations also limited the detection of pulsations at $405 \mathrm{GHz}$ (Figure 5). Nevertheless, it was possible to identify the presence of rapid pulsations in both events with qualitative amplitudes of about $\Delta S / S \approx 5 \%-10 \%$. 


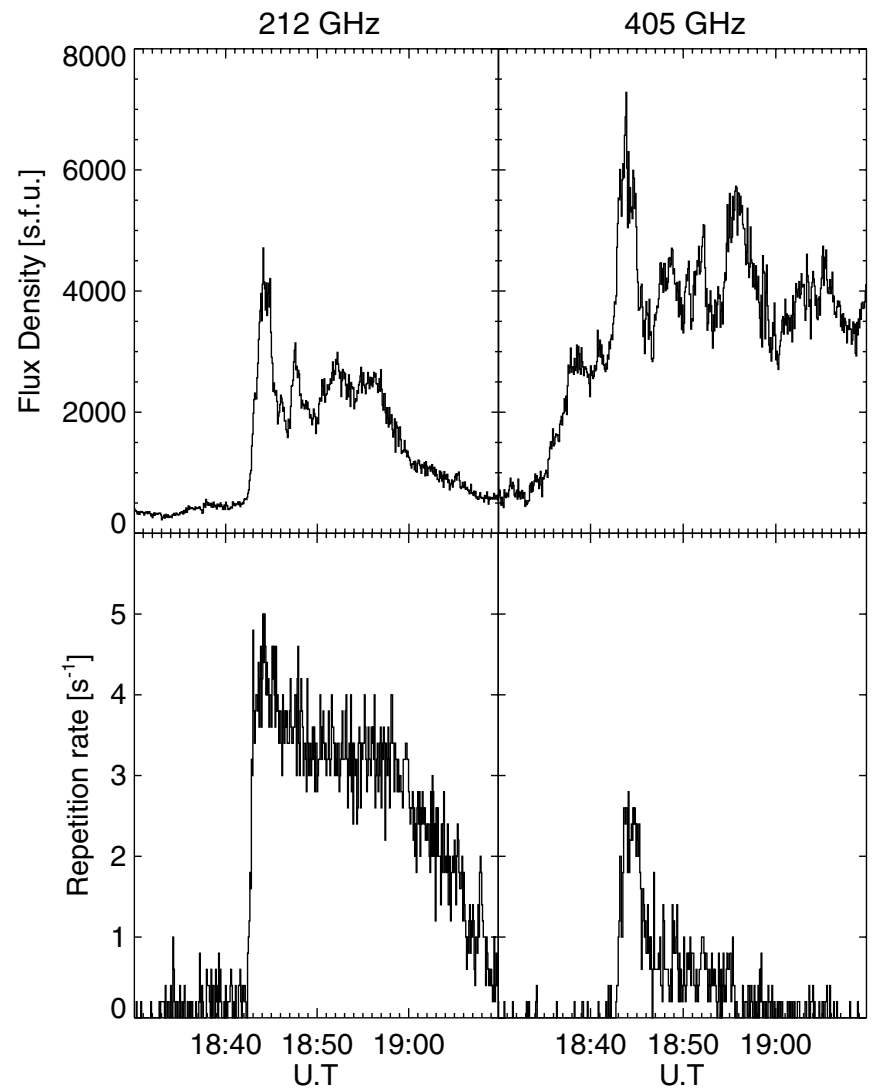

(a)

Figure 5. 2006 December 6 flare: (a) $212 \mathrm{GHz} 40 \mathrm{~ms}$ flux time profile (top), and pulse rate profile (bottom). (b) $405 \mathrm{GHz} 40 \mathrm{~ms}$ flux time profile (top), and pulse rate profile (bottom). Pulse rate obtained on $5 \mathrm{~ms}$ data, every $5 \mathrm{~s}$ interval, counted using wavelet decomposition. Due to relatively large atmospheric attenuation, most of the pulses at $405 \mathrm{GHz}$ were below the noise fluctuation threshold set prior to the event. They could be counted only partially in the main impulsive phase.

The results evidence a linear proportionality between the time profiles of pulse repetition rates with respect to the mean fluxes. The pulse repetition rate versus flux proportionalities is shown by the scatter diagrams of Figures 6-8, for the events of 2003 November 4 and 2 and 2006 December 6, respectively. The data scattering is more pronounced for 2003 November 2 and 2006 December 6, when atmosphere transmission was poor. Therefore, the suggested trend for clustering of points in groups might not be real and cannot be discussed.

Data for the 2003 November 4 event (Figures 3 and 6) are particularly meaningful, because they were obtained with the best atmosphere transmission conditions at the site. They provide very large values for the linear correlation coefficients of flux versus pulse repetition rates. The linear fits to the diagrams suggest a convergence to zero flux for zero pulse $\mathrm{s}^{-1}$. This trend may allow us to relate the solar burst sub-THz fluxes $S$ related to the superimposed pulse rates, $R$, as

$$
S=k R
$$

where $k$ has the dimension of energy (density per frequency band). The scatter diagrams shown in Figures 6-8 suggest that $k \approx 4000 \mathrm{SFU}$ s (i.e., $4 \times 10^{-19} \mathrm{~J} \mathrm{~m}^{-2} \mathrm{~Hz}^{-1}$ ) at $405 \mathrm{GHz}$, and $k \approx 800-1200$ SFU s (i.e., $0.8-1.2 \times 10^{-19} \mathrm{~J} \mathrm{~m}^{-2} \mathrm{~Hz}^{-1}$ ) at $212 \mathrm{GHz}$.

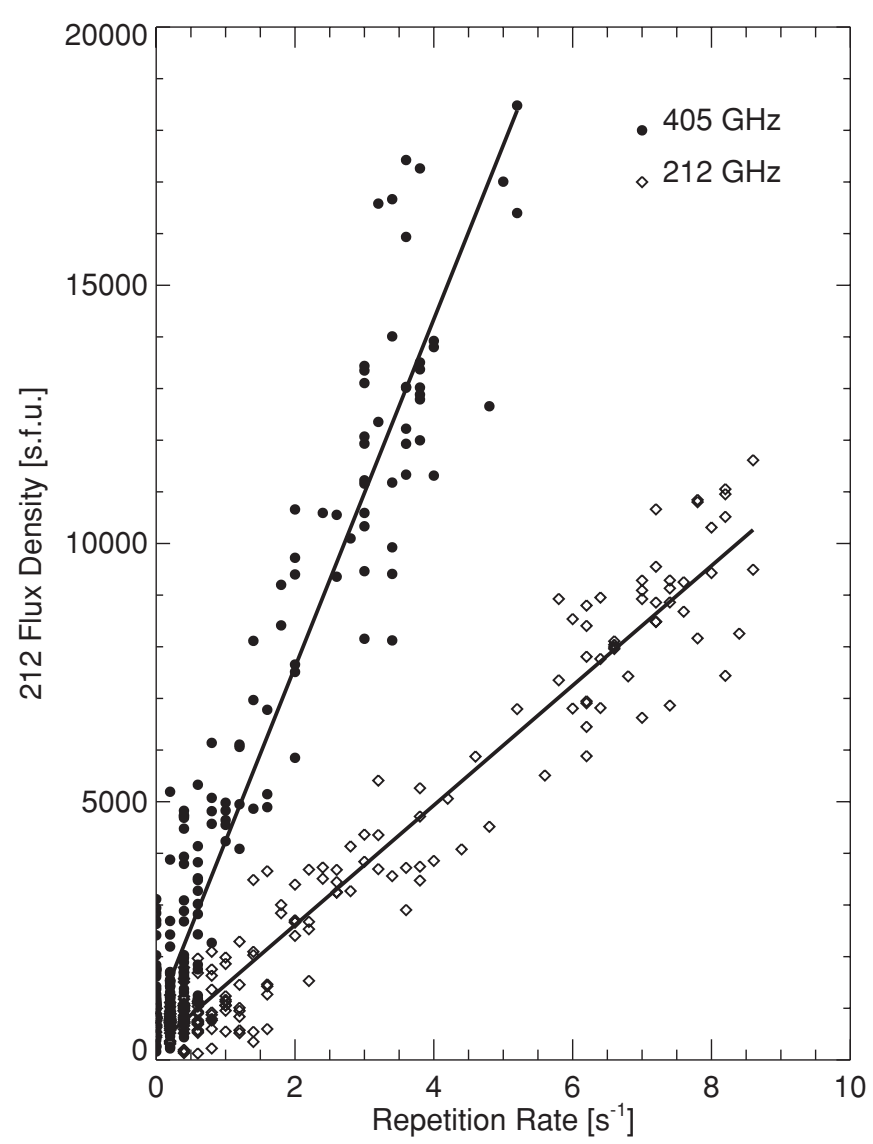

Figure 6. Scatter diagram showing the correlation between fluxes and pulse repetition rates for the 4 November 2003 event, at $212 \mathrm{GHz}$ (diamonds) and $405 \mathrm{GHz}$ (circles). The best-fit linear correlation coefficients are of 0.975 and 0.953 at 212 and $405 \mathrm{GHz}$, respectively.

\section{DISTINCTIONS OF RAPID PULSE FEATURES AT SUB-THz AND MICROWAVES}

The three events analyzed here had no microwave counterparts available for comparison with sufficiently high sensitivity and time resolution. However, for the 2003 November 4 burst, despite of saturation during the bulk of emission, the onset and decay phases were well observed at $44 \mathrm{GHz}$ by the Itapetinga radio telescope (Brazil), with high sensitivity and time resolution. Figure 9 shows the $100 \mathrm{~ms}$ flux time profiles at $44 \mathrm{GHz}$ (top) and the repetition pulse rate time profile (bottom).

A detailed comparison of sub- $\mathrm{THz}$ and $\mathrm{GHz}$ pulse time profiles is shown for the burst onset in Figure 10. The flux scale is the same for the three frequencies to illustrate that it is not possible to make a meaningful one-to-one pulse comparison in the two frequency ranges because the sub-THz pulse intensities are close to their smaller limiting values and about 20 times weaker compared to the $\mathrm{GHz}$ intensities. The $44 \mathrm{GHz}$ pulse amplitudes relative to the mean flux $\Delta S / S$ are about $80 \%$, nearly ten times larger than $\Delta S / S$ at the two sub-THz frequencies. It compares well to large $\Delta S / S$ values obtained for other bursts at $48 \mathrm{GHz}$ (Raulin et al. 1998). Comparing Figure 10 with Figure 3 we note that the onset of the $44 \mathrm{GHz}$ pulses begins about 2 minutes earlier than that of the sub-THz, which can be a consequence of the much larger amplitude of the $\mathrm{GHz}$ pulses. The pulse rates at 18:43 UT and at about 18:49 UT are of about $0.5-1.0$ per second, which is comparable for the sub$\mathrm{THz}$ and $\mathrm{GHz}$ frequencies during the periods of simultaneous observation. 


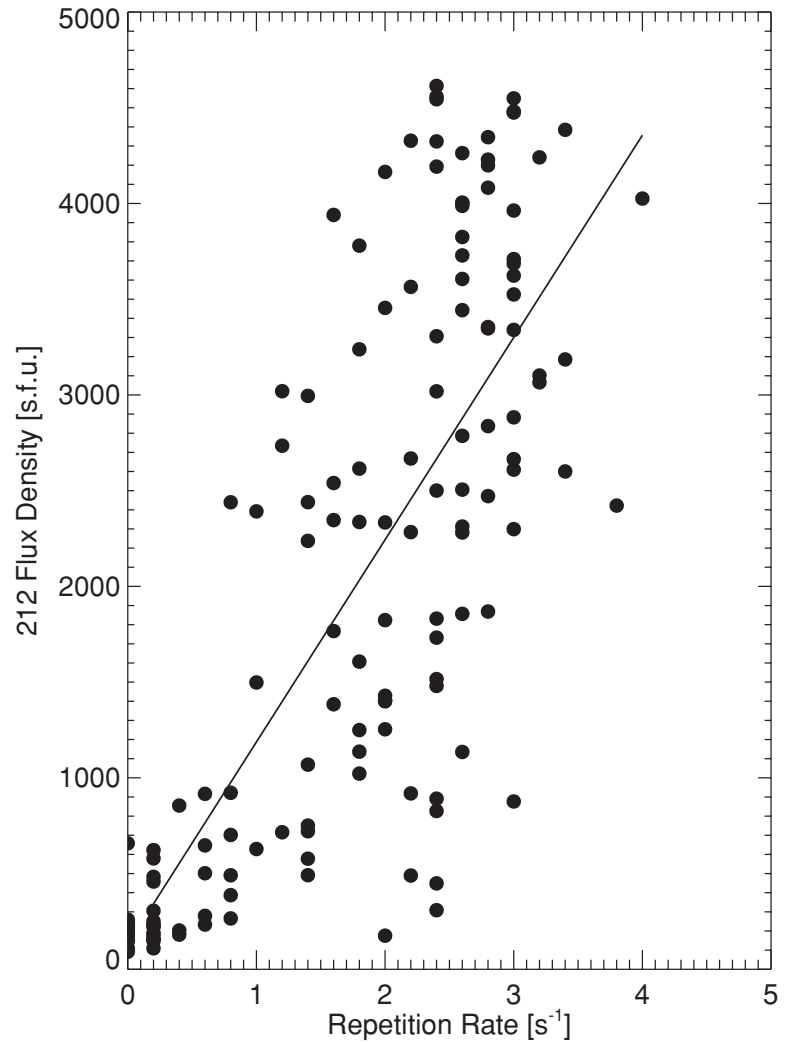

Figure 7. Scatter diagram showing the correlation between fluxes and pulse repetition rates for the 2003 November 2 event, at $212 \mathrm{GHz}$. Despite of the data scattering caused by the poor atmosphere transmission, the best-fit linear correlation coefficient is of 0.74 at $212 \mathrm{GHz}$.

The flux versus pulse rates relationship, $S \propto k R$, known at microwaves, shows a consistent qualitative trend on widely scattered data points obtained for various events (Kaufmann et al. 1980; Qin et al. 1996; Huang et al. 1996; Raulin et al. 1998). It has been known that the relative pulse amplitudes $\Delta S / S$ observed at discrete microwave frequencies range from few percent at about $10 \mathrm{GHz}$ up to $50 \%$ at $90 \mathrm{GHz}$, as shown in Figure 11 (plotted after Correia \& Kaufmann 1987, with new data added). This might provide a consistent explanation for the large pulse amplitude observed at $44 \mathrm{GHz}$ on 2003 November 4 burst. Unfortunately there were no high time resolution data available at any other microwave frequencies. On the other hand, there is a clear distinction in the sub-THz relative pulse amplitudes which are considerably smaller than the extrapolations (Correia \& Kaufmann 1987), as well as in comparison with the $44 \mathrm{GHz}$ pulses observed before and after saturation.

These results suggest that although there might exist a causal relationship between superimposed pulses observed at microwaves and at sub-THz bursts, they are probably produced at different physical locations and/or by different emission mechanisms.

\section{DISCUSSION}

The pulses superimposed onto the broadband solar bursts emission might be interpreted as a response to physical processes at the origin of the flare. Different mechanisms may be assigned for emissions producing the distinct spectral structures (Figure 1). Maximum microwave fluxes are at frequencies in the range 5-20 GHz (Guidice \& Castelli 1975), and some times extending up to $100 \mathrm{GHz}$ (Croom 1971; Nakajima et al. 1985;

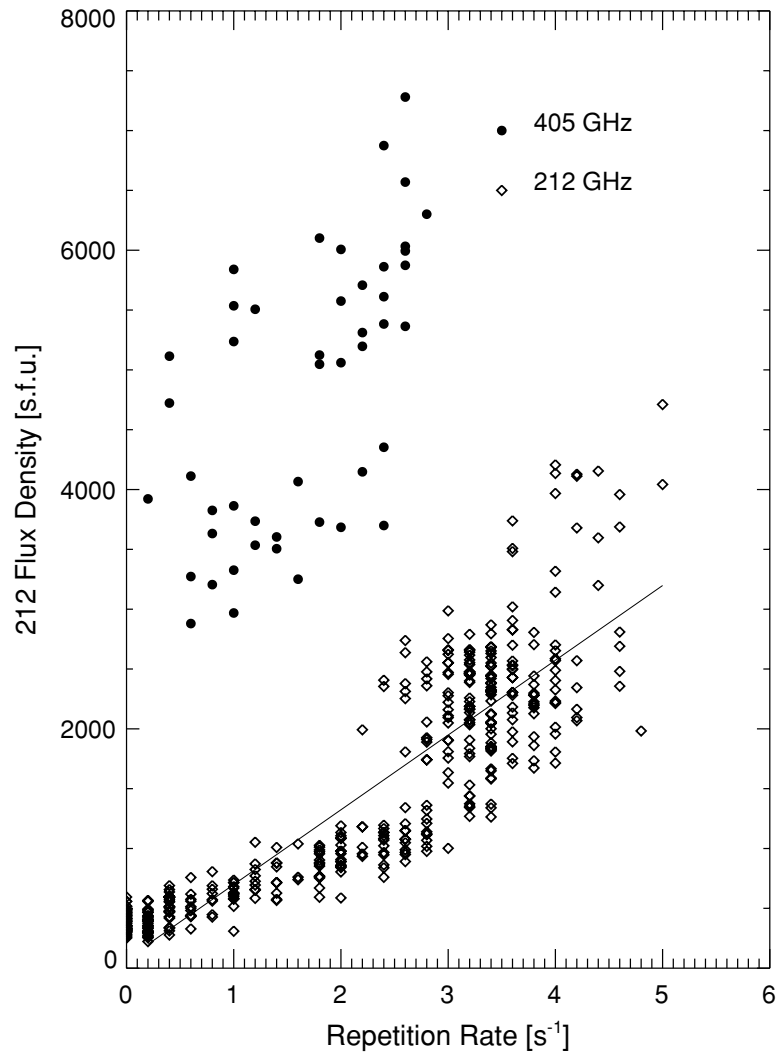

Figure 8. Scatter diagram showing the correlation between fluxes and pulse repetition rates for the 2006 December 6 event, at $212 \mathrm{GHz}$ (diamonds) and $405 \mathrm{GHz}$ (circles). Data scattering is due to fluctuation in poor atmospheric transmission. The best-fit linear correlation coefficient is of 0.87 at $212 \mathrm{GHz}$. The $405 \mathrm{GHz}$ data points are too scattered to be meaningful (correlation coefficient of 0.61 ), but they do indicate a qualitative trend, similar to the result shown in Figure 6.

Bastian et al. 2007). The spectral component B in Figure 1 is usually attributed to electron gyrosynchrotron radiation (Dulk 1985; Bastian et al. 1998). At meter-decimeter wavelengths the burst emission intensity increases for smaller frequencies (spectral structure A in Figure 1). This component is usually attributed to plasma emission excited by fast electrons or by shock waves (Wild \& Smerd 1972). The nature of the THz burst emission (structure $\mathrm{C}$ in Figure 1) is not well understood. It might be attributed to a distinct synchrotron emission by ultrarelativistic electrons (Kaufmann et al. 2004, 2009; Kaufmann \& Raulin 2006). Often the burst emission at the U-shaped minima (roughly $0.5-5 \mathrm{GHz}$, see Figure 1) may contain contributions of different origin added together (Allaart et al. 1990; Altyntsev et al. 2003). Mixed contributions from different mechanisms may also occur at the $30-200 \mathrm{GHz}$ spectral minimum.

Quasiperiodic pulsations of gyrosynchrotron emission at microwaves are known to cover a wide range of time scales-from hundreds of milliseconds to tens of seconds-and are interpreted in terms of modulation of source parameters undergoing MHD oscillations and/or modulation of electron acceleration or injection (Fleishman et al. 2008). The proportionality between flux and pulse rate at millimeter waves (Kaufmann et al. 1980; Raulin et al. 1998) supports the hypothesis that impulsive bursts might be a response to rapid injections quasiquantized in energy (Kaufmann et al. 1980; Sturrock et al. 1984). The ripple structure superimposed onto impulsive bursts time profiles might be the result of the convolution of pulses of a given lifetime with a variable repetition rate (Loran et al. 1985). 

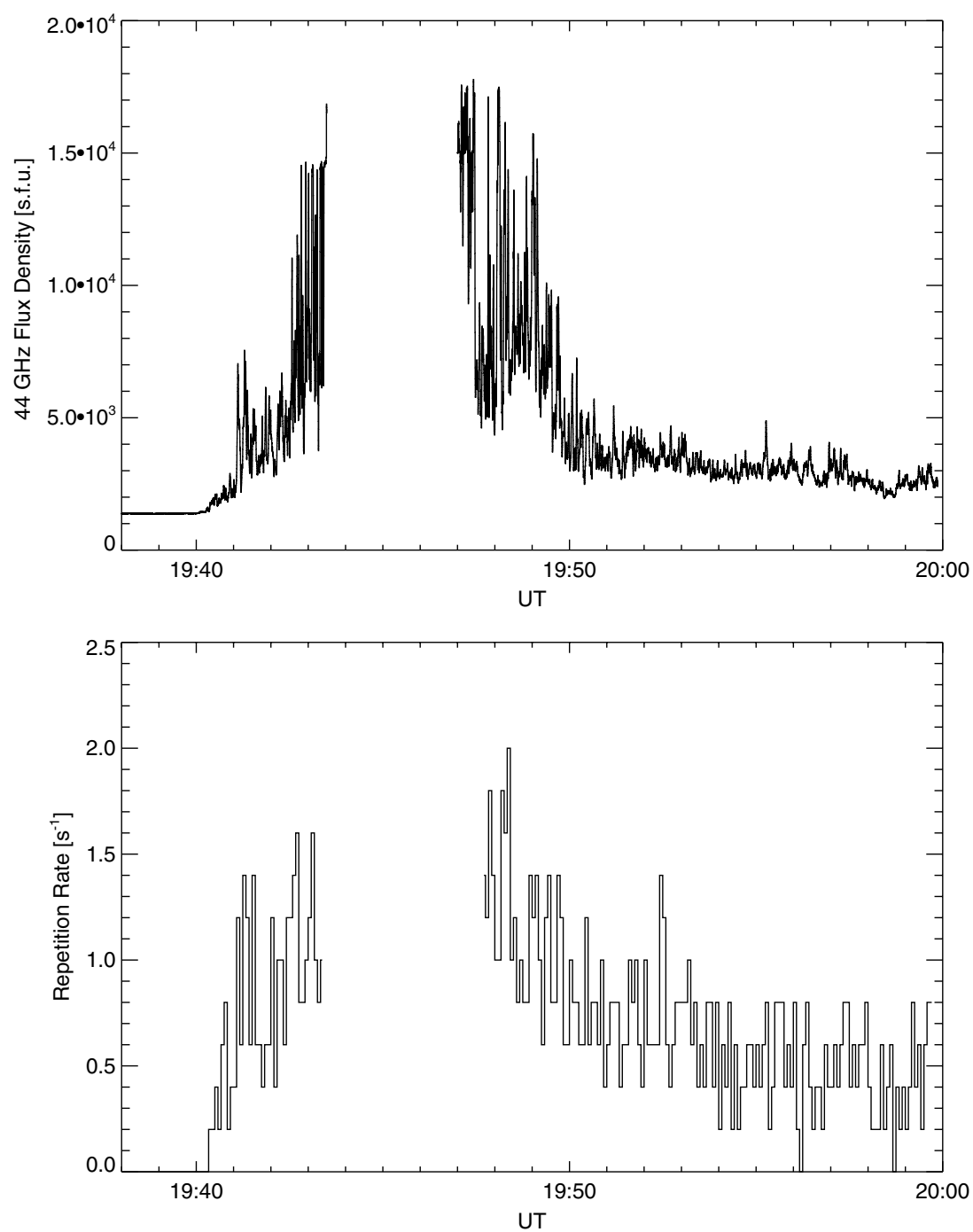

Figure 9. 2003 November 4 flare onset and decay phases at $44 \mathrm{GHz} 100 \mathrm{~ms}$ flux time profile (top), and a pulse rate profile (bottom). Pulse rates were obtained every $5 \mathrm{~s}$ interval, counted using wavelet decomposition.

The flux versus pulse rate correlation was also suggested to be a response to the inverse correlation between the saturation time of a wave-particle plasma instability originating the burst and the radiation time (Huang et al. 1996). This effect might also be attributed to the modulation of flare kernel emission by magnetic field oscillations driven by Alfvén waves, whose period decreases for larger temperatures (Qin et al. 1996). This interpretation assumes that the $\mathrm{GHz}$ emission is produced by gyro-synchrotron radiation from electrons moving in the active region magnetic field. However, Fleishman et al. (2008) interpretation of pulsating microwave burst (10-20 s duration pulses), based on Dulk (1985) gyro-synchrotron emission formulae, has shown that for sausage mode MHD oscillations the flux variations in the optically thick part of the spectrum (i.e., frequencies smaller than the spectral peak in Figure 1 structure B) should be anticorrelated in time with variations observed in the optically thin part of the spectrum (i.e., for frequencies higher than the spectral peak). They did not observe this effect, leading them to suggest that the pulsations were associated with quasiperiodic injection of emitting electrons.

It has been found qualitatively that the relative pulse amplitude $\Delta S / S$ increases for higher frequencies, as shown in
Figure 11 (Kaufmann et al. 1985; Correia \& Kaufmann 1987). This trend agree with the large relative pulse amplitudes observed at $44 \mathrm{GHz}$ (about $80 \%$ ) at the onset and decay of the 2003 November 4 event (see Figures 9 and 10) nearly ten times larger than the sub-THz pulses relative amplitudes. However, the observed sub-THz $\Delta S / S$ for the three events analyzed here are considerably smaller than expected when extrapolated from GHz observations (Kaufmann et al. 1985; Correia \& Kaufmann 1987; Figure 11).

The sub-THz component might be described as the optically thick part of the synchrotron radiation emitted by highly energetic electrons $(>10 \mathrm{MeV})$ moving in a strong magnetic field ( $\geqslant 1000$ Gauss; Kaufmann et al. 2004, 2009; Kaufmann \& Raulin 2006; Silva et al. 2007; Trottet et al. 2008; Cristiani et al. 2008). It should be somehow causally related but physically distinct from the $\mathrm{GHz}$ component. The linear relationship between sub-THz fluxes and pulse repetition rates might be a direct indication that the flare accelerator injects discrete quantized energy packets. The emissions at microwaves as well as at other ranges of energy might present proportional relations to these repetitive energetic injections that need more investigation. 


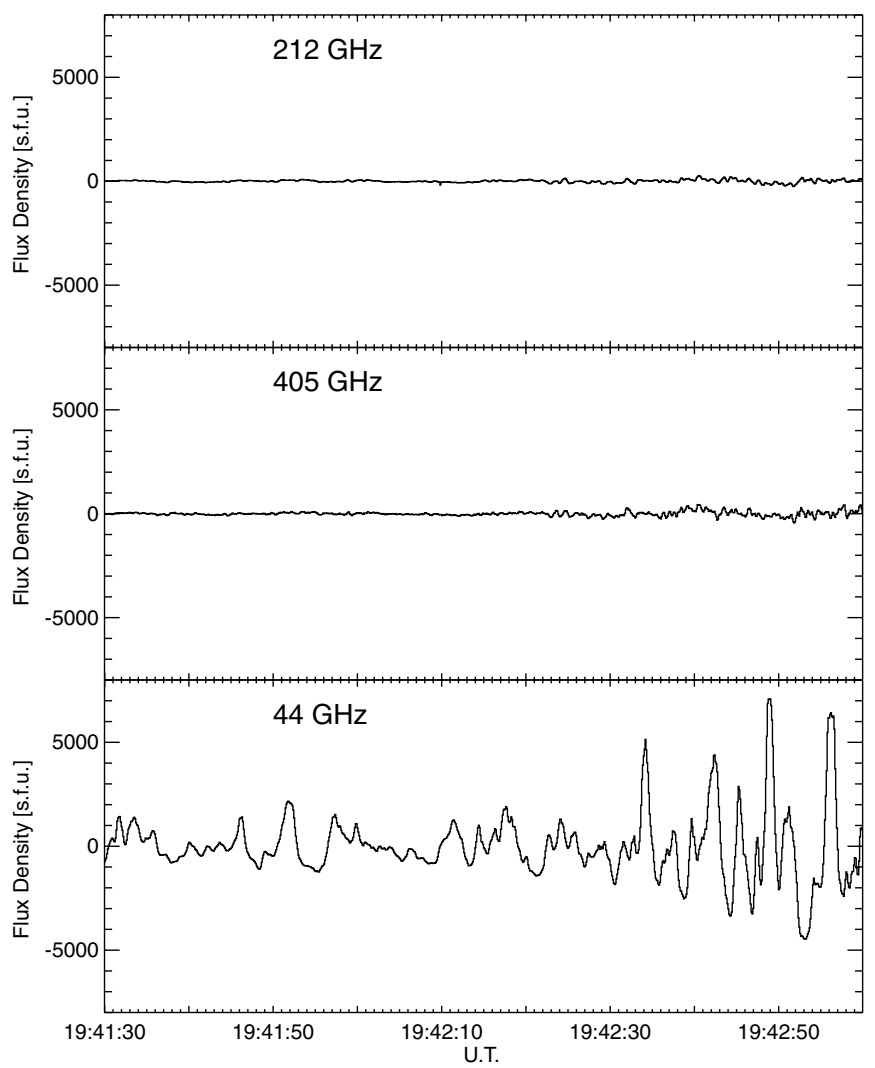

Figure 10. Expanded view of 2003 November 4 time profiles at the burst onset, just previous to the saturation at $44 \mathrm{GHz}$, illustrating how stronger were the $\mathrm{GHz}$ pulses as compared to the sub- $\mathrm{THz}$ pulses.

\section{CONCLUDING REMARKS}

It has been shown that rapid pulsations are found superimposed onto all events that present the $\mathrm{THz}$ spectral component. Pulse rate estimates were performed using wavelet decomposition at two sub-THz frequencies, 212 and $405 \mathrm{GHz}$. The pulse count criteria were set by fluctuation thresholds determined before the events. Pulses were particularly well defined for one event (2003 November 4). For the other two events, the pulse identification was limited by the poor quality of atmosphere transmission, affecting mainly the $405 \mathrm{GHz}$ data.

Similar small $\Delta S / S$ were found for two other solar flares observed at sub-THz frequencies, on 2001 April 6 (Kaufmann et al. 2002), and on 2001 August 25 (Raulin et al. 2003). They were not included in this study because there were indications that the sub- $\mathrm{THz}$ emissions might contain contributions from both $\mathrm{GHz}$ and $\mathrm{THz}$ spectral components (B and $\mathrm{C}$ in Figure 1).

Nevertheless, the analysis revealed two relevant properties of the sub-THz pulses: (1) the mean fluxes $(S)$ are proportional to the pulse repetition rates $(R)$ according to the simple relationship $S \approx k R$; (2) the pulse amplitudes relative to the mean flux, $\Delta S / S$ are small, ranging $5 \%-10 \%$ throughout the bursts durations. It follows that the flux versus pulse rate relationship might be interpreted as response to discrete and successive energetic injections, quantized in energy: $4 \times 10^{-19} \mathrm{~J} \mathrm{~m}^{-2} \mathrm{~Hz}^{-1}$ at $405 \mathrm{GHz}$, and $0.8-1.2 \times 10^{-19} \mathrm{~J} \mathrm{~m}^{-2} \mathrm{~Hz}^{-1}$ at $212 \mathrm{GHz}$.

The result (1) confirms the flux versus pulse rate qualitative relationship known in $\mathrm{GHz}$ bursts (Kaufmann et al. 1980; Qin et al. 1996; Huang et al. 1996; Raulin et al. 1998), and shows a good fit to a linear correlation. The correlation coefficients worsen when atmospheric transmission is poor, especially at

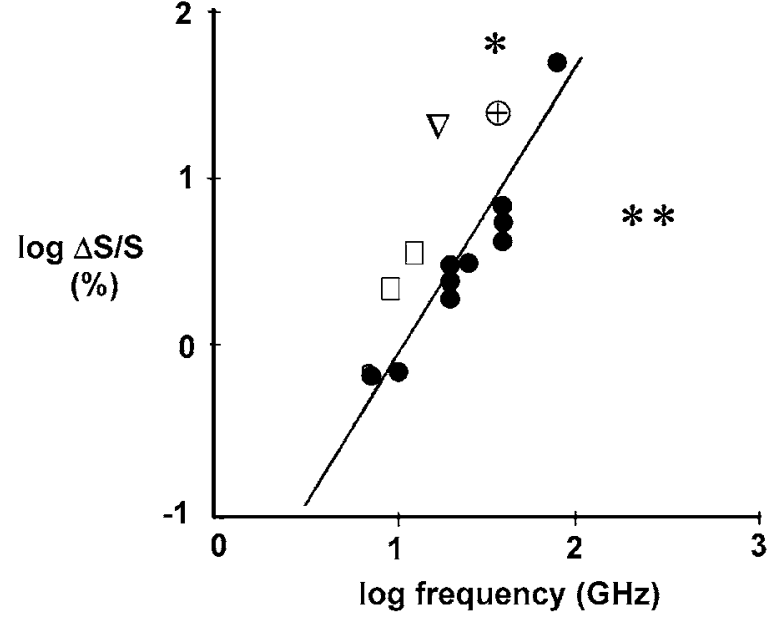

Figure 11. Qualitative scatter diagram showing the relative pulse amplitude $(\Delta S / S)$ dependence in frequency, after Correia \& $\operatorname{Kaufmann}(1987, \bullet)$, to which have been added data derived from Qin et al. (1996, $\square$ ), Huang et al. (1996, o), Raulin et al. $(1998, \oplus)$, and from Nakajima $(2000, \nabla)$. The 2004 November 4 data analyzed here are shown by the symbol $(*)$.

$405 \mathrm{GHz}$. The sub-THz flux increase with frequency might be associated with the optically thick part of synchrotron emission by high energy electrons $(>10 \mathrm{MeV})$.

In the $\mathrm{GHz}$ range, however, the relative pulse amplitude $(\Delta S / S)$ increases substantially with frequency (Correia \& Kaufmann 1987; Huang et al. 1996; see Figure 11), an effect that was confirmed by observations at $44 \mathrm{GHz}$ for the onset and late decay of the 2003 November 4 burst (Figures 9 and 10). For the sub-THz events, however, the observed $\Delta S / S$ are comparable at the two frequencies ( 212 and $405 \mathrm{GHz}$ ) and considerably smaller than expected from extrapolated values. This apparent qualitative contradiction might be an indication that two distinct emission mechanisms produce the spectrally separated $\mathrm{GHz}$ and $\mathrm{THz}$ components.

The production of the two $\mathrm{GHz}$ and $\mathrm{THz}$ spectral components (components B and C in Figure 1) might be explained by conceiving independent accelerators at the flaring source, producing different energy electrons nearly simultaneously, emitting the two spectral components. The spectral components B and $\mathrm{C}$ in Figure 1 would be representative of two synchrotron emission spectra, by electrons with mildly relativistic energies and ultrarelativistic energies, respectively. However the observations available are still too limited to support this possibility. Another scenario suggested by Wild \& Smerd (1972) might be adopted, placing a single accelerator closer to a single polarity foot-point injecting electrons into a magnetic morphology split into two separate loops, one low altitude with stronger field, another weaker field, higher above the solar surface, originating the two synchrotron spectral components, on peaking in the $\mathrm{GHz}$ and another in the $\mathrm{THz}$ range. Although this possibility was suggested in the discussion of the 2003 November 2 event (Silva et al. 2007), it requires very strong magnetic fields, of the order of the largest ones observed to date. In both scenarios, the relative pulse amplitude $(\Delta S / S)$ features in the $\mathrm{GHz}$ and sub$\mathrm{THz}$ ranges remain to be explained. An alternate interpretation take into account the contribution to spectral emissions from electron beam instabilities observed in laboratory accelerators (Nodvick \& Saxon 1954; Williams 2002; Carr et al. 2002; Byrd et al. 2002) proposed to happen in solar flares (Kaufmann \& Raulin 2006; Klopf 2008).

Observations are needed at higher frequencies in the $\mathrm{THz}$ range, as well as with higher time resolution and sensitivity in 
the $\mathrm{GHz}$ range. High sensitivity and time resolution solar patrol polarimeters at 45 and $90 \mathrm{GHz}$ are planned for El Leoncito Observatory with the support of the Brazilian research agency FAPESP and Argentinean agency CONICET. Solar photometry at two $\mathrm{THz}$ frequencies are considered by the experiment Detection of Solar eruptive Infrared Radiation (DESIR) on the French-China satellite SMall Explorer for the study of Solar Eruptions (SMESE; Vial et al. 2008). These new experiments are expected to bring new clues to understand the origin, the nature, and the relationship between flare emission at $\mathrm{GHz}$, $\mathrm{THz}$, and other ranges of energy, in response to repetitive discrete energetic injections, and their possible association with analogous physical processes in laboratory accelerators.

We gratefully acknowledge the remarks given by one anonymous referee helping the improvement of this paper presentation. These researches were partially supported by the Brazilian agencies FAPESP, CNPq, Mackpesquisa, and Argentina agency CONICET.

\section{REFERENCES}

Allaart, M. A. F., et al. 1990, Solar Phys., 130, 183

Altyntsev, A. T., et al. 2000, Solar Phys., 195, 401

Altyntsev, A. T., et al. 2003, A\&A, 411, 263

Aschwanden, M. J., et al. 1998, ApJ, 505, 941

Bastian, T. S., Benz, A. O., \& Gary, D. E. 1998, ARA\&A, 36, 132

Bastian, T. S., Fleishman, G. D., \& Gary, D. E. 2007, ApJ, 666, 1256

Bendjoya, P., Petit, J.-M., \& Spahn, F. 1993, Icarus, 105, 385

Benz, A. O., et al. 1992, A\&A, 36, 131

Butz, M., et al. 1976, Kleinheubacher Ber., 19, 345

Byrd, M., et al. 2002, Phys. Rev. Lett., 89, 224801

Carr, G. L., et al. 2002, Nature, 420, 153

Castelli, J. P. 1972, in CESRA Conf. Proc. 3, ed. J. Delanoy \& F. Poumeyrol (Bordeaux-Floirac: CESRA), 69

Cornell, M. E., et al. 1984, ApJ, 279, 875

Correia, E., \& Kaufmann, P. 1987, Solar Phys., 111, 143

Costa, J. E. R., \& Kaufmann, P. 1983, A\&A, 119, 131

Cristiani, G., et al. 2008, A\&A, 492, 215

Croom, D. L. 1971, Solar Phys., 19, 152

Dulk, G. A. 1985, ARA\&A, 23, 169
Dulk, G. A., Melrose, D. B., \& White, S. M. 1979, ApJ, 234, 1137

Fleishman, G. D., Bastian, T. S., \& Gary, D. E. 2008, ApJ, 684, 1433

Gaizauskas, V., \& Tapping, K. F. 1980, ApJ, 241, 804

Giménez de Castro, C. G., et al. 1999, A\&A, 140, 343

Giménez de Castro, C. G., et al. 2001, A\&A, 368, 317

Guidice, D. A., \& Castelli, J. P. 1975, Solar Phys., 44, 155

Huang, G., Qin, Z., \& Yao, Q. 1996, A\&AS, 243, 401

Kaufmann, P., \& Raulin, J.-P. 2006, Phys. Plasma, 13, 070701

Kaufmann, P., et al. 1980, A\&A, 87, 58

Kaufmann, P., et al. 1983, Solar Phys., 84, 311

Kaufmann, P., et al. 1985, Nature, 313, 380

Kaufmann, P., et al. 2000, Solar Phys., 197, 361

Kaufmann, P., et al. 2001, ApJ, 548, L95

Kaufmann, P., et al. 2002, ApJ, 574, 1059

Kaufmann, P., et al. 2004, ApJ, 603, L121

Kaufmann, P., et al. 2008, Proc. SPIE, 7012, 7012L

Kaufmann, et al. 2009, Solar Phys., in press

Klopf, J. M. 2008, in 1st SMESE Workshop, Paris, France, March 10-12 (Paris: Inst. d'Astrophys. de Paris), http://www.ias.u-psud.fr/smese/main_1st.psp

Krüger, A., et al. 1994, Astron. J. Suppl., 90, 683

Kundu, M. R., \& Vlahos, L. 1982, Space Sci. Rev., 32, 405

Kurths, J., \& Schwarz, U. 1994, Space Sci. Rev., 68, 171

Lim, J., et al. 1992, Solar Phys., 140, 343

Loran, J. M., et al. 1985, Solar Phys., 97, 363

Lüthi, A., Lüdi, A., \& Magun, A. 2004, A\&A, 420, 361

Makhmutov, V. S., et al. 2003, Solar Phys., 218, 211

Nakajima, H., et al. 1985, PASJ, 37, 163

Nakajima, H. 2000, in ASP Conf. Ser. 206, High Energy Solar Physics: Anticipating HESSI, ed. R. Ramaty \& N. Mandzhavidze (San Francisco, CA: ASP), 313

Nita, G. M., Gary, D. E., \& Lee, J. 2004, ApJ, 605, 528

Nodvick, J. S., \& Saxon, D. S. 1954, Phys, Rev., 96, 180

Qin, Z., et al. 1996, Solar Phys., 163, 383

Raulin, J.-P., et al. 1998, ApJ, 498, L173

Raulin, J.-P., et al. 2003, ApJ, 592, 580

Raulin, J.-P., et al. 2004, Solar Phys., 223, 181

Silva, A. V. R., Wang, H., \& Gary, D. E. 2000, ApJ, 545, 1116

Silva, A. V. R., et al. 2007, Solar Phys., 245, 311

Sturrock, P. A., et al. 1984, Solar Phys., 94, 341

Takakura, T., et al. 1983, Nature, 302, 317

Trottet, G., et al. 2008, ApJ, 678, 509

Vial, J.-C., et al. 2008, Adv. Space Res., 41, 183

Wild, J. P., \& Smerd, S. F. 1972, ARA\&A, 10, 159

Williams, G. P. 2002, Rev. Sci. Instrum., 73, 1461 